\title{
Community-Lab: Overview and Invitation to the Research Community
}

\author{
Leandro Navarro ${ }^{\dagger}$, Pau Escrich ${ }^{\ddagger}$, Roger Baig ${ }^{\ddagger}$, Axel Neumann* \\ *Pangea.org, Barcelona, Spain \\ ${ }^{\dagger}$ Universitat Politècnica de Catalunya - BarcelonaTECH, Barcelona, Spain \\ ${ }^{\ddagger}$ Guifi.net, Catalonia, Spain
}

\begin{abstract}
Community-Lab is an open, distributed infrastructure for researchers to experiment with Community Networks, that are large scale, self-organized and decentralized networks and services built and operated by citizens for citizens. The goal of Community-Lab is to advance research and empower society by understanding and removing obstacles for these networks and services. This article outlines Community-Lab's aim, development, characteristics and infrastructure. This demonstration presents its current status of development and deployment, a sample decentralized experiment, and an invitation to the broader large-scale distributed computing research community to participate in the project through an open call.
\end{abstract}

\section{INTRODUCTION}

The Internet connects the world. However, providing sustainable, cost-effective and quality services with coverage for all citizens is still challenging. Community networking, also known as "bottom-up networking", is an emerging model for the Future Internet where communities of citizens build, operate and own open IP-based networks, a key infrastructure for individual and collective digital participation. Hundreds of community networks operate in rural, urban, rich or poor areas. These networks are usually run by non-profit organizations and can cooperate with local stakeholders to develop community services including local networking, voice, data, Internet access.

These "community networks" are large-scale distributed and decentralized system with many computing nodes, links, content, services and traffic. They are extremely dynamic and diverse as they are built in a decentralized manner mixing wireless and wired (optical) links with diverse routing schemes with many services and applications. There are no barriers for the participation as governance, knowledge and ownership is open, with an open peering agreement governing the network. Therefore these networks are not just decentralized but also self-owned and self-managed by community members, selfgrowing in links, capacity and services provided. These large, decentralized, dynamic and heterogeneous structures raises challenges that the research community can address.

\section{Community-LAB}

The Community-Lab ${ }^{1}$ testbed is being developed and operated by the CONFINE project ${ }^{2}$ by federating existing community IP networks. This testbed supports experimentallydriven research on community-owned open local IP networks.

\footnotetext{
${ }^{1}$ Community-Lab portal: http://community-lab.net

${ }^{2}$ Confine: FP7 Integrated Project (2011-2015) http://confine-project.eu
}

It integrates with and extends three existing community networks: Guifi.net (Catalonia, ES), FunkFeuer (Vienna, AT) and AWMN (Athens, GR); each in the range of 500-20,000 nodes, a greater number of links and even more end-users. Fraunhofer (DE), IBBT (BE) and UPC (ES) contribute in research, Pangea (ES) operates the testbed and the OPLAN Foundation (UK) coordinates dissemination.

Simulations or small controlled laboratory experiments can produce results that are hard to translate to real scenarios. Similarly to Planetlab in academic networks, CommunityLab aims at providing researchers a realistic environment for experimentation with the characteristics (for the best and worst) of real community networks. The testbed is operational, with more than 40 nodes in several communities in Europe before end of 2012 and growing over the next three years.

The ultimate goal for research in community networks is to explore the opportunities and remove obstacles for community networks in terms of scalability and sustainability. We expect that this research will directly impact the quality of community networks by improving current shortcomings and providing a sustainable model of community networking.

\section{RELATED RESEARCH TESTBEDS}

There are other related testbeds ${ }^{3}$. The Berlin RoofNet project is an experimental mesh network in Berlin consisting of nearly 50 indoor nodes mainly to provide students with Internet access as well as VoIP. The IBBT w-iLab.t is a sensor network with 200 indoor locations. The NITOS testbed has around $40 \mathrm{WiFi}$ nodes in a single building for small scale controlled experiments. Finally, the ORBIT testbed, an indoor grid of around 400802.11 radio nodes, is the largest indoor wireless testbed to date.

Community-Lab is aiming at providing a realistic environment, thus not reproducibility-oriented, with traffic sent through real community links and routed on real community networks participating in the testbed. Experiments in Community-Lab are fundamentally different from running them in an indoor (laboratory) or controlled environment or on an isolated wide-area testbed. Its value is to give researchers access to (1) a large set of nodes and links from different community networks; (2) a realistic network that experiences congestion, failures, and diverse link and node behaviors; and (3) the potential participation of the community with data or testing experimental services.

\footnotetext{
${ }^{3}$ berlinroofnet.de, ilabt.ibbt.be, nitlab.inf.uth.gr, orbit-lab.org
} 


\section{The COMmUnity-LAB InFRASTRUCTURE}

Community-Lab is an open, distributed infrastructure where researchers can deploy experimental services, perform experiments or access to open data traces. The testbed is inspired by PlanetLab but differs in two main ways: first, the domain is community networking: a large number of modest devices, limited network links more fragile and less managed than commercial or academic networks; and second, CommunityLab is designed to allow experiments ranging from the physical layer, routing, transport, applications and services, or even social experiments. These differences result in different choices and features as described below but a high-level architecture equivalent to Planetlab's.

Hardware and network connectivity: Community-Lab consists of at least one portal or controller and a set of nodes that are embedded in different community networks. A Community-Lab node consists of two or three devices: the community device, the research device and an optional recovery device connected together by a wired local network, with the community device acting as a gateway.

This separation tries to preserve the stability and setup of the community network by allowing the connection of relatively powerful research devices (e.g. x86 Atom boards with at least 1 GB RAM) to existing community devices.

Software: It follows the abstraction in PlanetLab of a slice as a set of separate virtual machines called slivers and a virtualized network. This allows resource sharing and isolation among experiments. Unlike PlanetLab, CommunityLab can provide a separate public IP address to each sliver and a separate virtual network to avoid conflicts with other experiments or the community traffic. The Community-Lab software is free open-source software so that other similar testbeds can be setup.

Research devices run a customized OpenWrt firmware which allows simultaneously running several slivers implemented as Linux containers with controlled access to the device's resources to ensure slice and network isolation. This is guaranteed by the control software. It provides researchers with a familiar Linux environment with root access and rich connectivity options including: simple NAT access like many home computers, public IP addresses to serve incoming connections, layer 2 access to VLAN-tagged networks to support disruptive experiments such as routing, traffic capture with anonymization, and limited raw physical link access.

Administration and access: Community-Lab uses an operation model similar to PlanetLab, and in the near future it will federate with other SFA compliant testbeds. Researchers interested in running experiments should contact the support team to apply for access. The CONFINE consortium coordinates research on the testbed, and it even provides funding for selected experiments (see VI).

\section{EXPERIMENTAL NETWORK AND SYSTEMS RESEARCH}

Community-Lab is open for research on a wide range of problems relevant to community networks. The challenges stem from a combination of scale and heterogeneity; the need for decentralized management mechanisms for large, diverse and volatile resources; interdependency across typically separate layers given the limited resources; dynamics requiring adaptive mechanisms for continuous changes, selfhealing due to node or link failures, self-optimization or even self-protection from attacks.

Adaptive decentralized algorithms and systems are required to run these large-scale dynamic and heterogeneous distributed systems that are the network and services provided by community networks. Examples of research topics: wireless channel allocation, hybrid optical-radio networks, mesh routing, cross-layer optimizations, self-management, quality of service, decentralized overlays, incentives and economics, social networks, content distribution, socio-technical evaluation, security and privacy, performance modeling.

\section{THE DEMONSTRATION}

The demonstration at the P2P12 conference is a live demo of Community-Lab with three key aspects: 1) A live demo of Guifi.net, with more than 17,000 nodes, with an operational community device connected to guifi.net using an outdoor antenna and a wireless (WiFi) link. The demo shows a live visualization of the network graph, links, and a routing map; 2) A live demo of Community-Lab with an operational research device connected to the community device and the testbed web portal; 3) An experiment evaluating BitTorrent implementations running on the testbed.

Therefore the demonstration is composed by three computers: one community device (Guifi.net), one research device, and a computer controlling the experiment and the visualization. A visitor can browse through the network map and live data. The presentation describes how the testbed works and the opportunities for the research community to get involved, particularly through the CONFINE open call for experiments.

The demo can help researchers to visualize and understand community networks and the research challenges. As the testbed can be accessed remotely the demo can serve to discuss ideas for experiments. Researchers can be supported by an open call for experiments offered by the CONFINE project (around 50,000 EUR funding per experiment, deadline by mid October 2012).

\section{CONCLUSIONS}

Three major characteristics make Community-Lab unique: 1) large scale with an expected size of several hundreds of nodes; 2) integration with existing community networks; 3) support for long running experiments by means of node and network virtualization.

The demo helps explain the research challenges in community networks and how Community-Lab can be used for experimentally-driven research in the coming three years, supported by the CONFINE project. The open call for experiments is an invitation to the research community to be involved.

Experimental research results can help develop an inclusive Future Internet where being "connected" is more a human right and a community asset for social development rather than just a service for private profit and public consumption.

\section{Acknowledgments}

This work is supported by the CONFINE Integrated Project 288535. Special thanks to the participants in the CONFINE project, and to everyone involved in the community networks. You are real wizards! 